following areas; procedures, emergencies, prioritising and outpatient work.

From the results above we can ascertain that consultants and SHO's have answered similarly with regards their ability/ preparation to perform for the skills of clinical examination and history taking. However, they answered the questions on procedures, teamwork and out-patient work significantly differently.

Conclusion There is a discrepancy between consultant opinion of ability and SHO's feeling of preparedness for some of the same skills. The reason for this difference in opinion should be explored further in future studies.

SHO's have identified areas of initial weakness which they feel could have been improved by better preparation. More work, focusing on these specific aspects of undergraduate paediatric education needs to be done to improve graduates preparedness for the role of paediatric SHO.

\section{P255 BUILDING RESUSCITATION CAPABILITY IN LOW RESOURCE SETTINGS; DEVELOPMENT OF THE PAIRS COURSE (OUTLINING THE DEVELOPMENT OF A TAILORED PAEDIATRIC RESUSCITATION PROGRAMME FOR LOW RESOURCE HEALTHCARE SETTINGS)}

${ }^{1}$ Rachel MacDonell ${ }^{*},{ }^{2}$ Stanley Koe, ${ }^{3}$ Ikechukwu Okafor, ${ }^{4}$ Adam James, ${ }^{3}$ Claire Ahern, ${ }^{5}$ Trish Scanlan. ${ }^{1} R C P I$, Dublin, Ireland; ${ }^{2}$ Tallaght University Hospital, Dublin, Ireland; ${ }^{3}$ Children's University Hospital, Dublin, Ireland; ${ }^{4}$ Our Lady's Children's Hospital, Dublin, Ireland; ${ }^{5}$ Muhimbili National Hospital, Dar Es Salaam, Tanzania, United Republic of

\subsection{6/archdischild-2019-epa.605}

We have developed and tested a dynamic, context-specific paediatric resuscitation course with tiered level of complexity with targeting different levels of healthcare workers in limited resource healthcare settings. The PAIRS course is now established in Dar Es Salaam, Tanzania with a plan for spread to Uganda and Nigeria in 2019.

In 2012, under a joint initiative between the Paediatric Oncology Unit at Muhimbili National Hospital and the Tanzanian children's cancer charity, Their Lives Matter (TLM), a team of Irish paediatric resuscitation specialists was invited to train the oncology staff. This early enterprise has matured into provision of an annual training programme by a dedicated paediatric faculty, with course content specifically designed for use in a limited resource setting and expanded to involve clinical staff from any specialty, with interest from all paediatric settings and from adult, maternity and neonatal specialties.

Children on the paediatric oncology ward were experiencing poor clinical outcomes after common oncological emergencies and inter-current illnesses. Deterioration was sometimes slow to detect with $100 \%$ mortality rates for any child requiring CPR. Multifactorial issues were identified including:

- Poor team approach to resuscitation

- Low knowledge of basic and advanced life support

- Non-availability of emergency equipment

- No access to paediatric intensive care support

- Additional hurdles related to culture, communication and infrastructure

Established internationally recognised resuscitation courses were trialled over the years, but none was deemed appropriate for the setting.
The one-day Paediatric Assessment of Illness, Recognition and Resuscitation (PAIRS) course was designed to build foundational skills and knowledge of any nurse or doctor working with children in a limited resource setting. A further one-day PAIRS $^{+}$module was developed for paediatric doctors and senior nurses.

The three-pronged implementation strategy consists of

1. a massive rollout of the course, targeting the entire paediatric community of the hospital, championed by local nursing and medical management

2. local endorsement and accreditation

3. establishment of local faculty and national accreditation of the course with the aim of handing over to the local faculty after 5 years of sustained annual training.

A variety of engagement strategies are necessary to influence behaviour and drive change. In order to achieve active buy-in for sustainability, it is essential to engage with local stakeholders, foster cooperative relationships and adapt, adopt or generate training programmes that reflect the real-life needs of the clinical team and setting are critical success factors that influence sustainability, scale and spread.

\section{P256 SCHOOL GATES - TOBACCO AND SMOKE FREE ZONES}

Claire Duffy*. NIPC, GALWAY, Ireland

\subsection{6/archdischild-2019-epa.606}

Background Smoking is the leading cause of preventable death in Ireland. 5,200 smokers die every year. 8\% of Irish $10-17$ year olds smoke. Tobacco Free Ireland has set a target of < $5 \%$ smoking prevalence by 2025 . $80 \%$ of smokers start before they reach 18 years of age and they become addicted very quickly. $19 \%$ of under 25 -year olds smoke. Tobacco free policies inside and outside all education settings and at school gates will reduce smoking rates.

Aim The aim of this project was to examine the evidence to support smoke free schools and, explore ways of promoting a smoke free environment both within and outside the school gate parameter.

Results Smoke free policies help to educate the community about environmental tobacco smoke. Smoking has effects on the pregnant mother and the unborn child. Cigarette smoke trigger asthma, glue ear and chest infections. Liquid nicotine containers are appealing to children and may be lethal if ingested. Smoking is more prevalent in schools without antismoking policies. Enforcement of school tobacco policies is associated with fewer observations of tobacco use by minors on school grounds as well as lower rates of current smoking among students. Properly implemented school programs to prevent tobacco use and addiction can lower smoking prevalence by up to $60 \%$. School-based tobacco use prevention programs are largely effective for most tobacco use related outcomes. Comprehensive tobacco-free policies are effective in reducing exposure and intention to smoke on campus. Noncollege educated young adults smoke at twice the rate of college-educated adults.

Evaluation National campaigns relating to smoking cessation both nationally and internationally were reviewed. 'A Health Promoting School can be characterised as a school which is constantly strengthening it's capacity as a healthy place in which to live, learn and work.' 